\title{
ASSESSMENT OF MITRAL VALVE DISEASE BY LEFT HEART CATHETERIZATION
}

\author{
BY \\ ERNEST W. HANCOCK* \\ From the Cardiac Department, Guy's Hospital, and the Thorndike Memorial Laboratory and the Second and Fourth \\ Medical Services (Harvard), Boston City Hospital, and the Department of Medicine, Harvard Medical School, Boston, \\ Massachusetts \\ Received October 24, 1958
}

Many studies of the pressures in the left atrium and the left ventricle of patients with mitral valve disease have been published. Initially obtained by direct puncture of the cardiac chambers at operation, such recordings have since been made possible by the development of techniques of left atrial puncture as a diagnostic procedure before operation. Direct determinations of the left atrial pressure, the left ventricular diastolic pressure, the diastolic pressure gradient across the mitral valve, and the duration of the diastolic filling period render unnecessary several assumptions that must be made when mitral disease is assessed by right heart catheterization. When mitral stenosis is associated with aortic valve disease, left ventricular failure, or significant mitral regurgitation, the limitations of right heart catheterization may be great, and precise information may be obtainable only by left heart catheterization.

Assessment of the relative importance of mitral stenosis and regurgitation when present in combination has remained a difficult problem. Various opinions of the diagnostic value particularly of the left atrial pulse form (Gunning and Linden, 1958; Marshall et al., 1957; and Morrow et al., 1957) and of dye dilution curves (Korner and Shillingford, 1955; Shillingford, 1958; and Woodward et al., 1957) have been expressed. The present report summarizes experience with left heart catheterization in 75 patients with mitral valve disease, with particular reference to the assessment of combined stenosis and regurgitation.

\section{MeThodS}

Left atrial puncture was carried out by the right posterior transthoracic route (Biork et al., 1953) in the prone position. A thin-walled cannula of $1.5 \mathrm{~mm}$. outer diameter, containing a short-bevelled needle, was used. The left ventricle was catheterized with a polyethylene catheter of $0.90 \mathrm{~mm}$. internal diameter. Simultaneous left atrial and left ventricular pressures were recorded in many cases by means of a second left atrial needle. Pressures were recorded with strain gage (Statham P23D) or capacitance electromanometers (Southern Insts. Ltd.) on a direct writing oscillograph (Sanborn) or a mirror galvanometer photographic recorder (New Electronic Products Ltd.). Pressures were referred to a zero level at mid-thorax.

The mitral diastolic pressure gradient, defined as the mean difference between left atrial and left ventricular pressure during the diastolic filling period, was measured on superimposed pressure tracings, either as directly recorded (Fig. 1-2) or as redrawn. A significant mitral diastolic pressure gradient has been defined as a gradient equivalent to at least $2 \mathrm{~mm}$. $\mathrm{Hg}$ throughout the diastolic filling period. If left atrial and left ventricular pressures became equalized during diastole, there has been considered to be no significant gradient, although such patients sometimes showed a small gradient for the initial 0.04-0.08 sec. of diastole (Fig. 8A).

Mitral valve area was calculated from a modification of the formula of Gorlin and Gorlin (1951). Diastolic filling period was measured directly on superimposed left atrial and left ventricular pressure tracings, rather than on the brachial arterial tracing, and the directly measured mitral diastolic pressure

* Research Fellow of the American Heart Association, and Research Fellow, Cardiac Department, Guy's Hospital. 389 


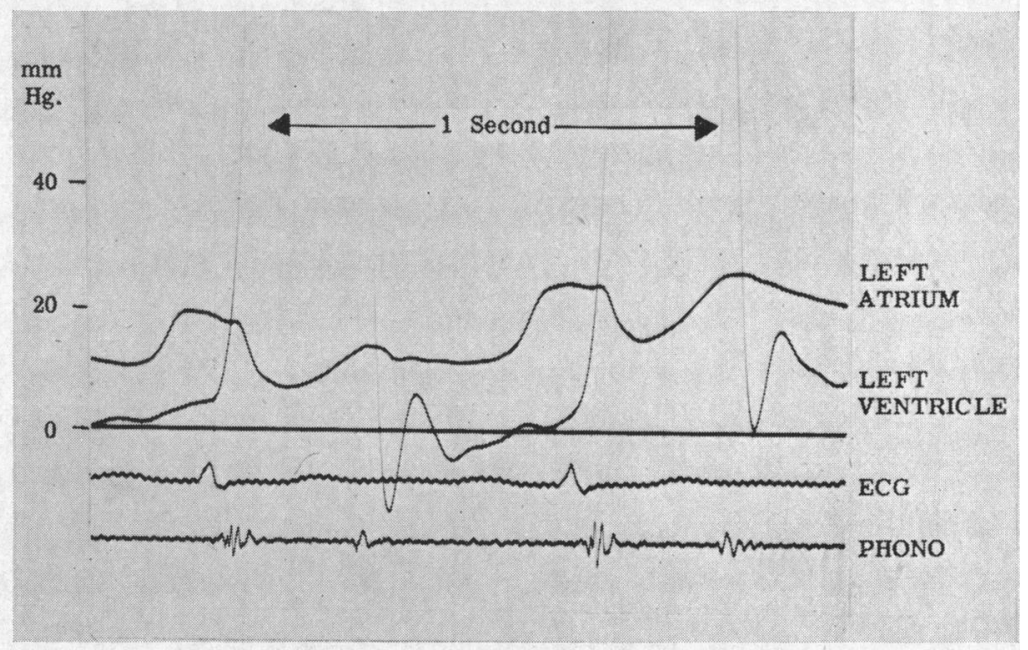

Fig.1.- Left atrial and left ventricular pressures, electrocardiogram, and phonocardiogram recorded simultaneously in a patient with pure mitral stenosis and normal rhythm. Mitral diastolic gradient $16 \mathrm{~mm}$. $\mathrm{Hg}$, heart rate $86 /$ min., cardiac output $4.01 . / \mathrm{min}$., and calculated mitral valve area 0.5 sq. $\mathrm{cm}$.

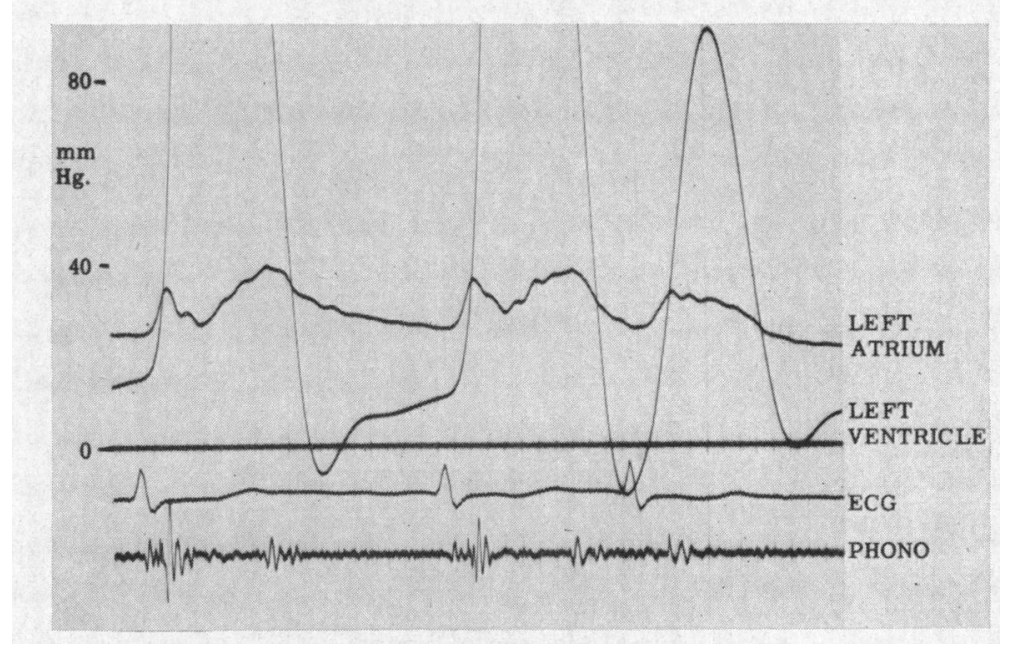

FIG. 2.-Left atrial and left ventricular pressures, electrocardiogram, and phonocardiogram recorded simultaneously in a patient with pure mitral stenosis and atrial fibrillation. Mitral diastolic pressure gradient $21 \mathrm{~mm}$. $\mathrm{Hg}$, heart rate $100 / \mathrm{min}$., cardiac output $5 \cdot 2 \mathrm{l} / \mathrm{min}$., calculated mitral valve area $0.4 \mathrm{sq} . \mathrm{cm}$. Vertical divisions $=0.04 \mathrm{sec}$.

gradient was used rather than the pulmonary wedge pressure minus 5 . An empirical constant of 44.5 was used. The formula thus became:

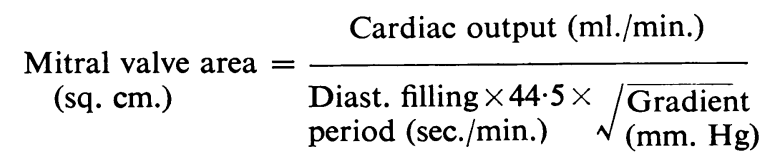


Mitral valve area at operation was determined by measurement of drawings made by the surgeon. In general, valves $1 \mathrm{~cm}$. in length and admitting only a finger tip had areas of $1.0 \mathrm{sq} . \mathrm{cm}$. or less. Valves $1-2 \mathrm{~cm}$. in length, admitting a finger to the first joint with difficulty, were usually $1.0-1.5 \mathrm{sq} . \mathrm{cm}$., and valves admitting a finger freely were greater than $1.5 \mathrm{sq} . \mathrm{cm}$. The regurgitant jet at operation was judged $1+$ if only a fine localized jet, $2+$ if a considerable stream but primarily felt only at the orifice, $3+$ if a more powerful stream palpable widely in the atrium, and $4+$ if very gross. The last category was reserved for the two patients operated on for pure mitral regurgitation.

Cardiac output was determined in most cases by the dye dilution technique. Multiple samples were taken from the brachial artery at intervals of two seconds following injection of Evans Blue dye into the left atrium.

Technique and Complications. After initial trials of right and left lateral positions, the prone position has been used exclusively. This is well tolerated even by patients who are severely orthopnœic when supine. Barbiturate, meperidine, or morphine premedication is used in addition to local anæsthesia as deep as the pericardium. The appropriate intercostal space, usually the ninth, is located fluoroscopically as a final preliminary, and the needle is passed to the left atrium as described by Biork. A characteristic pulsatile sensation is always felt when the needle comes against the atrium, and no other guidance is needed. Inadvertent puncture of the right atrium requires reinsertion of the needle in a more medial direction. The left atrium has been successfully punctured in every case.

The left ventricle has been successfully catheterized in all but three, with no failures in the last 60 consecutive cases. This is facilitated by direction of the needle caudally as well as medially. Experience has shown that 15-20 minutes of manipulation with constant oscilloscopic observation of the pressure wave form results in successful catheterization of the left ventricle in almost every case.

There have been no deaths attributable to the procedure, and no instance of cardiac tamponade. Slight bleeding from the left atrium occurs, as sero-sanguinous pericardial fluid is found at subsequent operation in the great majority. Injury to the right lung, usually by the preliminary local anæsthetic needle, has resulted in pneumothorax in eight and hæmoptysis in eight cases: the pneumothorax would usually go undetected were not a chest X-ray taken as a routine immediately after the procedure. Neither of these complications has ever been severe enough to require treatment. There has been only one instance of each in the last 35 cases.

Mild pleuritic pain in the right anterior chest for 12-24 hours is a usual side effect. Severe pain is very uncommon. Clinical evidence of pericarditis has not been seen, although in three patients in whom operation was delayed for 6-12 weeks after the procedure, a vascular adhesive pericarditis of apparently recent origin was found.

Arterial embolism has never occurred during the procedure, but has occurred in two cases, 3 and 5 days later. Both patients had established atrial fibrillation, one with multiple emboli previously. The embolus, to the superior mesenteric artery, was fatal in one: the other had a successful embolectomy from the aortic bifurcation, but died suddenly of unknown cause two weeks later. At autopsy, both had mural thrombus in the left atrial appendage, but none at the site of puncture, which was scarcely visible.

Atrial fibrillation began during the procedure in one of 20 patients with normal rhythm, and began the following day in two others. All three had a simultaneous right heart catheterization. Ventricular fibrillation or arrest has not occured.

\section{Classification of Patients}

The patients have been divided into three groups, according to the nature of the mitral valve lesion: (1) pure mitral stenosis, 35 cases, (2) combined mitral stenosis and regurgitation, 24 cases, and (3) pure mitral regurgitation, 14 cases. Patients with a significant gradient across the valve and no regurgitation clinically or at operation were included in group 1. Patients with a significant gradient across the valve and also regurgitation were included in group 2, which thus included some with predominant stenosis and some with predominant regurgitation. Patients have been included in group 3 if mitral regurgitation was diagnosed clinically, and no significant pressure gradient found across the valve. Two patients with clinical signs of pure mitral stenosis but no significant pressure gradient are considered separately.

The presence of a significant diastolic pressure gradient across the mitral valve, as previously defined, appears to be diagnostic of at least a certain degree of anatomical mitral stenosis. It 
probably implies a valve area of $3 \mathrm{sq}$. $\mathrm{cm}$. or less. Three of the patients found to have no gradient have later come to autopsy, and each has had a valve area of at least $3.5 \mathrm{sq} . \mathrm{cm}$., the orifice admitting three fingers (Fig. 3). Thus no evidence has been found that mitral regurgitation is associated with a significant diastolic gradient unless there is also some degree of stenosis.

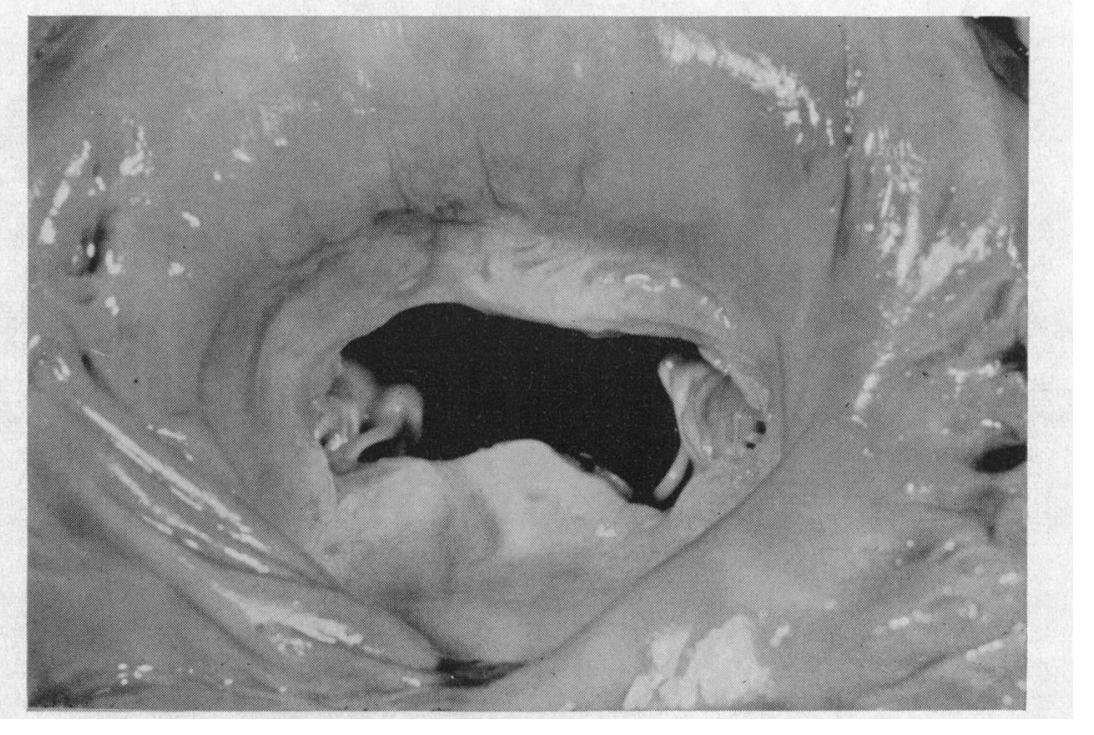

FIG. 3.-The mitral valve of a patient with pure mitral regurgitation. There was no diastolic pressure gradient across the valve. At operation, for mitral regurgitation, a gross $(4+)$ regurgitant jet was present.

\section{Assessment of the Degree of Stenosis}

The calculated valve area in pure mitral stenosis correlated closely with that observed at operation (Fig. 4) and there were no discrepancies of clinical importance. The gradient across the valve was not nearly so well correlated with the degree of stenosis, and the left atrial pressure even less well. Thus, the desirability of simultaneous determination of cardiac output with the pressure measurements, to allow calculation of the mitral valve area, is reaffirmed. The necessity of flow measurement is particularly great when the gradient is small, as this may be due to mild stenosis with a normal or high flow, or to severe stenosis with a low flow.

In combined stenosis and regurgitation, assessment of the degree of stenosis is less certain because of the unknown factor of regurgitant flow. As this cannot be measured, the mitral valve area has been calculated simply from the cardiac output, as in pure stenosis. In the majority of patients with combined stenosis and regurgitation in whom there is anatomical confirmation, the calculated valve area proved to be a reasonably accurate prediction (Table I). This suggests that regurgitant flow is only a small fraction of the cardiac output in these patients. Indeed, the degree of stenosis in this group was in the same range as in those with pure stenosis, even though clinical signs of regurgitation were often very prominent, and the regurgitant jet at operation $2-3+$. Three patients, however, proved to have valve areas more than twice the predicted size (Fig. 4). They must have had predominant regurgitation, and a much greater backflow than the other 11, so that use of the systemic cardiac output alone in calculating valve area underestimated the actual forward flow across the mitral valve and hence underestimated the valve size.

Thus, in patients with the clinical signs of mitral stenosis and regurgitation, investigated with present techniques of left heart catheterization, a division may first be made into (1) those with no pressure gradient across the valve, therefore having mitral regurgitation without significant stenosis, 


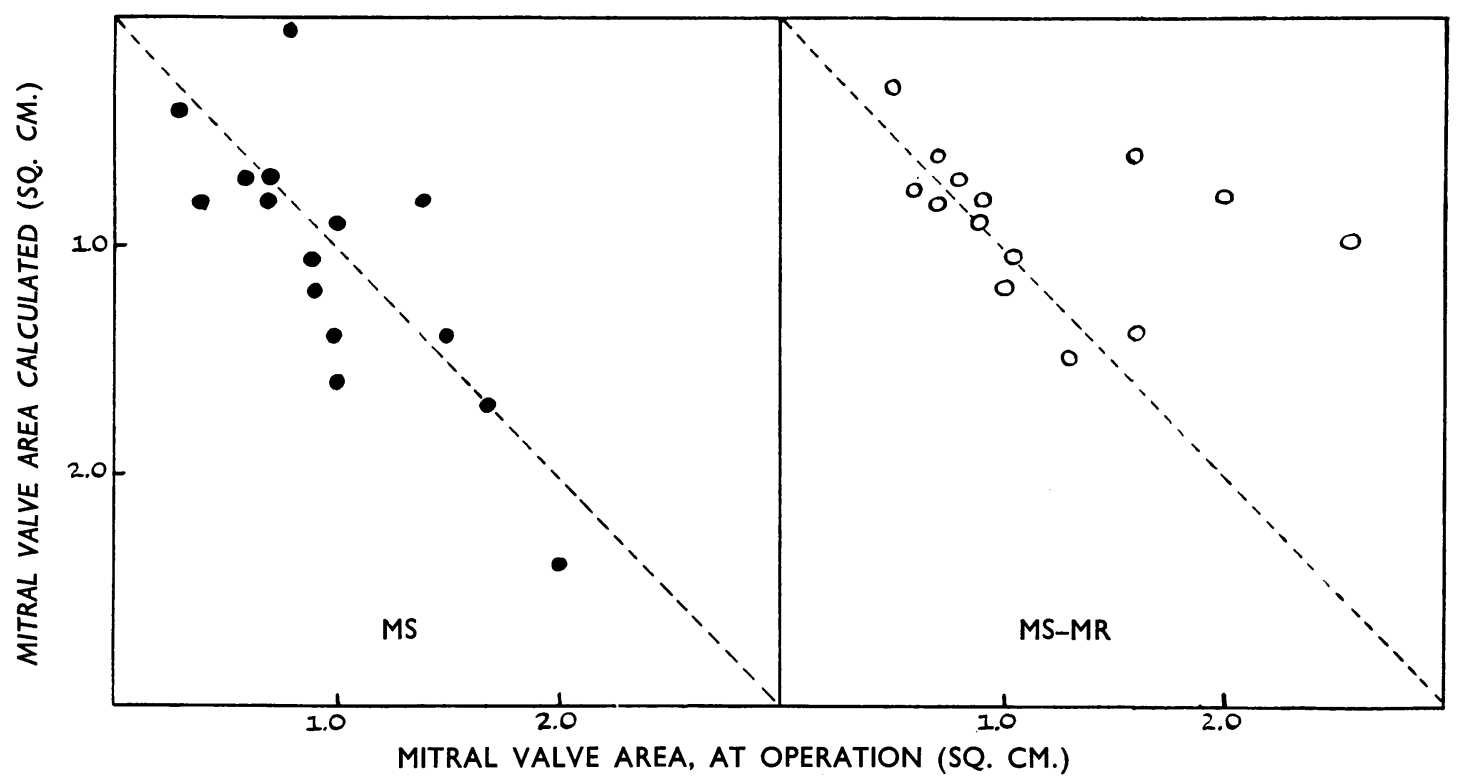

FIG. 4.-Calculated mitral valve area, on the ordinate in sq. $\mathrm{cm}$., and mitral valve area observed at operation, on the abscissa in sq. cm. Pure mitral stenosis, solid circles, on the left, and combined mitral stenosis and regurgitation, open circles, on the right. Mitral valve area calculated from the systemic cardiac output only, without regard for regurgitant flow.

TABLE I

Comparison of Catheterization and Operation or Autopsy Findings in Patients with Combined Mitral STENOSIS AND REGURGITATION

\begin{tabular}{l|c|c|c|c}
\hline & \multicolumn{2}{|c|}{$\begin{array}{c}\text { Mitral valve area, } \\
\text { sq. cm. }\end{array}$} & $\begin{array}{c}\text { Regurg. } \\
\text { jet at } \\
\text { operation }\end{array}$ & $\begin{array}{c}\text { Downslope of } \\
\text { dye dilution } \\
\text { curve. (sec.) }\end{array}$ \\
\cline { 2 - 3 } & \multicolumn{2}{|c|}{ Calc. } & Obs. & \\
\hline V.B. & 0.9 & 0.9 & ++ & 9.2 \\
H.H. & 1.5 & 1.3 & ++ & 6.2 \\
E.P. & 1.2 & 0.9 & ++ & 12.2 \\
M.R. & 0.7 & 0.6 & ++ & 18.2 \\
M.W. & 1.2 & 1.0 & (PM) & 10.1 \\
L.M. & 0.6 & 0.8 & ++ & 18.4 \\
E.H. & 0.8 & 0.7 & ++ & 16.2 \\
A.S. & 0.3 & 0.5 & (PM) & 28.8 \\
A.N. & 1.0 & 1.0 & ++ & 7.8 \\
M.B. & 1.2 & 1.0 & + & 13.6 \\
E.C. & 1.4 & 1.6 & +++ & 19.5 \\
A.M. & 1.2 & 2.5 & +++ & 13.1 \\
J.S. & 0.6 & 1.7 & +++ & 41.6 \\
I.J. & 0.8 & 2.0 & +++ & 51.8 \\
\hline
\end{tabular}

and (2) those with a significant gradient, who have a combination of stenosis and regurgitation. In the latter group, calculation of the mitral valve area on the basis of the systemic cardiac output will yield in most cases a reasonably accurate prediction of the degree of stenosis, but in a few cases, the regurgitant flow will be so large that the valve size will be significantly underestimated. In any case, the calculated valve area may be considered a minimal valve size, potentially an underestimate according to the degree of regurgitation judged to be present. For example, if the calculated valve area is 0.4 sq. $\mathrm{cm}$., it is evident that stenosis is severe, as the valve size would still be less than 
1 sq. cm. if regurgitant flow exceeded the forward output. On the other hand, if the calculated valve area is $2.5 \mathrm{sq}$. cm., it is obvious that stenosis is not severe whatever the regurgitant flow.

In spite of the limitation imposed by inability to measure regurgitant flow, left heart catheterization has been of much value in assessing patients with combined stenosis and regurgitation. Of 33 patients with this clinical problem in the present group, nine proved to have no gradient across the valve, 10 had calculated valve areas in excess of $1.5 \mathrm{sq} . \mathrm{cm}$. and have not been operated on, and 14 have been operated upon for mitral stenosis on the basis of a substantial pressure gradient and a calculated valve area less than $1.5 \mathrm{sq} . \mathrm{cm}$. The findings in the patients confirmed at operation are shown in Table I. Only three showed a disappointingly slight degree of stenosis at operation, and even in these valvotomy was considered worthwhile by the surgeon.

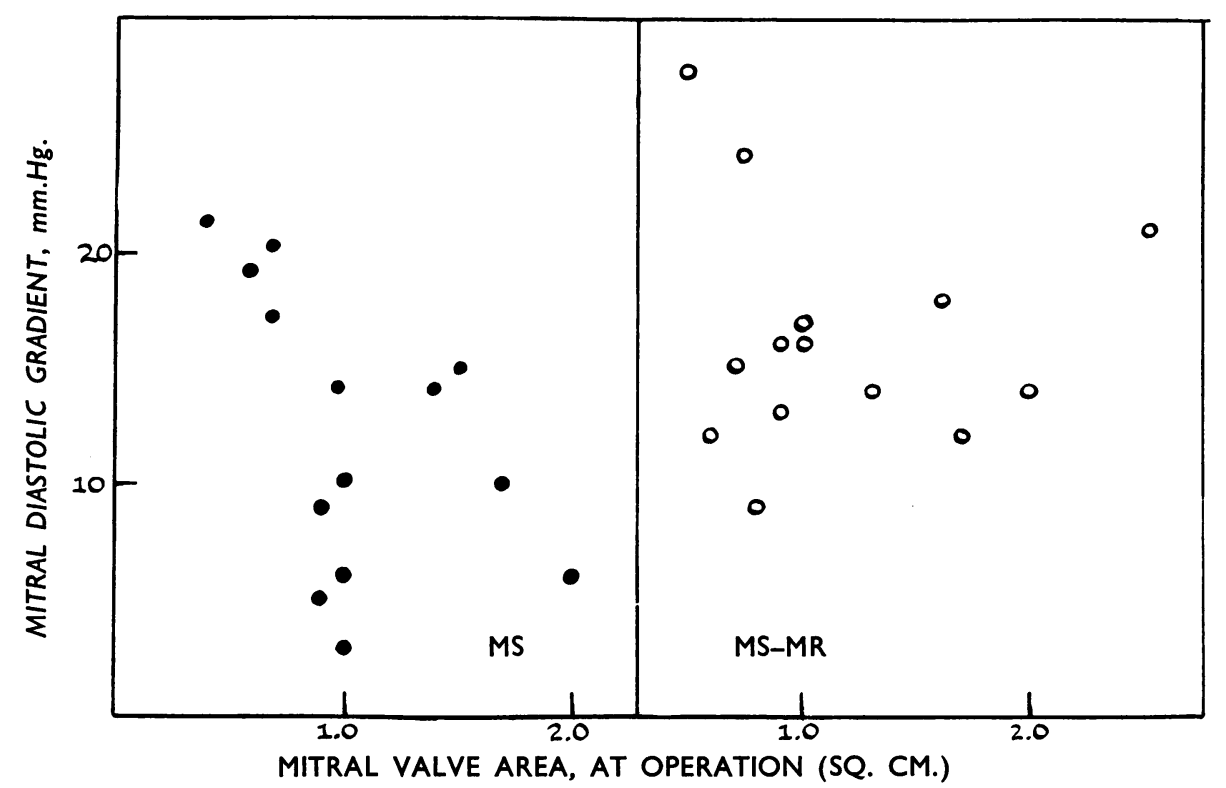

FIG. 5.-Mean mitral diastolic pressure gradient in $\mathrm{mm}$. $\mathrm{Hg}$ on the ordinate; mitral valve area observed at operation in sq. $\mathrm{cm}$. on the abscissa. Pure mitral stenosis, solid circles, on the left; combined stenosis and regurgitation, open circles, on the right.

The concept of predominant stenosis or predominant regurgitation is difficult to apply to some patients who seem to have a very significant degree of both. A patient with a mitral valve area of $2.5 \mathrm{sq}$. cm. has, at the most, moderate stenosis. Yet, regurgitation may so increase the flow across the valve as to require a pressure gradient of $15-20 \mathrm{~mm}$. $\mathrm{Hg}$. The resulting elevation of left atrial pressure, and the necessary further rise with effort, tachycardia, etc. is a hæmodynamic fault identical with that of tight mitral stenosis. While surgical relief of the stenosis alone may be beneficial, such a valve is generally much less favourable than one with tight stenosis. While the decision to operate for mitral stenosis involves many considerations, the presence of anatomically severe stenosis is obviously one of the most important. It is not enough merely to demonstrate a high left atrial pressure or a substantial gradient across the valve. A calculation of the valve area and some estimate of the degree of regurgitation are required in order to make the best possible prediction of the actual valve size.

\section{Left Atrial Pulse Form}

Many ways of analyzing the left atrial pulse form have been proposed to distinguish predominant stenosis from predominant regurgitation. Most of these are based on the fact that in mitral regurgitation the systolic $v$ wave is higher, the systolic $x$ descent less prominent, the early diastolic $y$ descent more rapid, and the diastolic and mean pressure lower than in mitral stenosis (Fig. 6). 


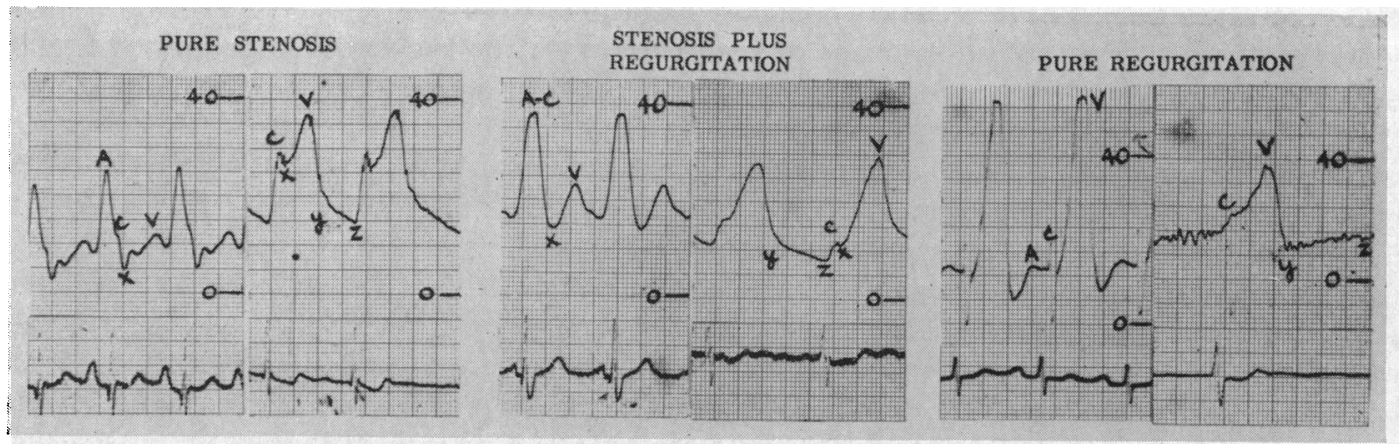

Fig. 6.-Typical left atrial pulse form in mitral valve disease; normal rhythm on the left and atrial fibrillation on the right of each pair.

In the present study, no single criterion or ratio reliably separated the three groups previously defined, or any two groups from one another. In general, there are distinctive differences in the left atrial pulse forms of patients with no significant gradient across the valve as opposed to those with a gradient. The diastolic portion of the tracing is the most useful. Of those patients with atrial fibrillation, the left atrial pressure showed a continuous fall throughout diastole in 33 of 38 patients with a significant gradient across the valve, whereas in 15 of 16 patients without a gradient, left atrial pressure remained the same or rose during diastole. It is this phenomenon that in a damped and delayed form is reflected in the $R y / V$ ratio in the pulmonary wedge tracing (Owen and Wood, 1953), and is a useful guide to the presence or absence of mitral obstruction, i.e. a gradient across the valve. It does not, however, necessarily distinguish predominant stenosis from predominant regurgitation.

The systolic $x$ descent was present in the majority of patients in all groups. As the deflection was less than $3 \mathrm{~mm}$. $\mathrm{Hg}$ in most of those with pure mitral stenosis, it was difficult to recognize impairment of the $x$ descent as a feature of regurgitation. A large systolic wave with no discernible $x$ descent was a sign of regurgitation, however, occurring in 5 of 11 patients with pure regurgitation, 2 of 20 with combined stenosis and regurgitation, and none of 19 with pure stenosis, considering only those with atrial fibrillation. Only one of these 7 patients had predominant stenosis.

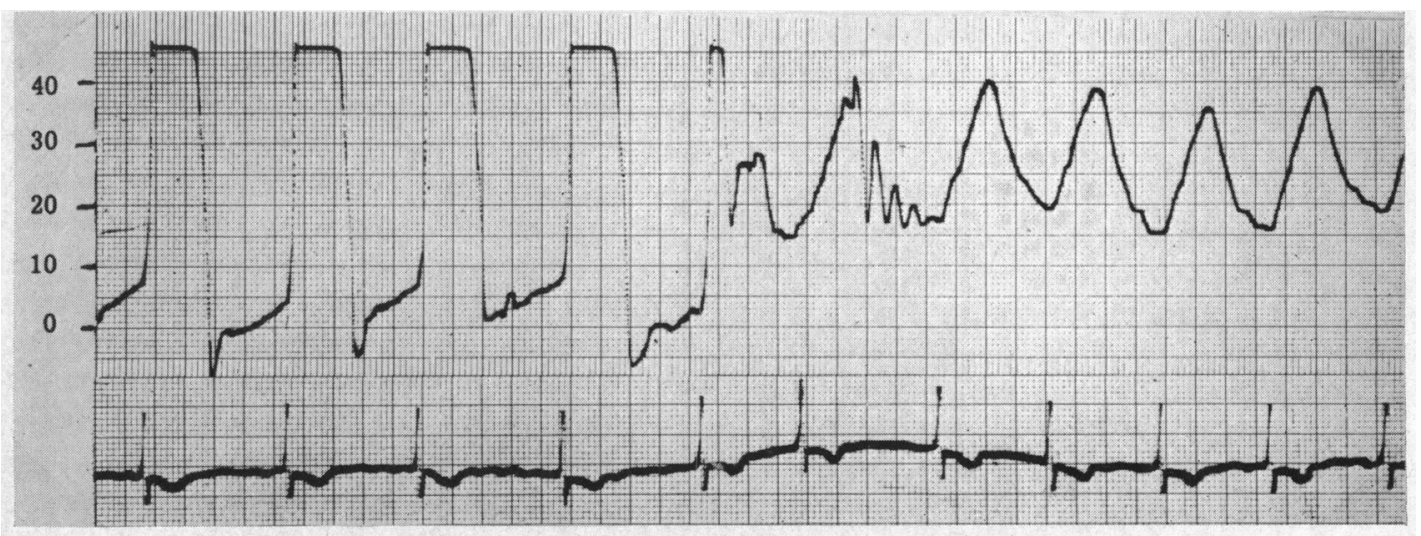

Fig. 7.-Left atrial and left ventricular pressures recorded consecutively in a patient with combined mitral stenosis and regurgitation. Mitral diastolic pressure gradient $13 \mathrm{~mm}$. $\mathbf{H g}$, cardiac output $4.41 . / \mathrm{min}$., calculated mitral valve area $0.6 \mathrm{sq}$. cm. Mitral valve area at operation $1.7 \mathrm{sq}$. cm., regurgitant jet $3+$. 
Of the three patients who were found at operation to have severe regurgitation with only moderate stenosis, two showed evidence of regurgitation in the left atrial pulse form, i.e. a large positive systolic wave with no $x$ descent in one (Fig. 7), and flat pressure during diastole in the other.

\section{Assessment of Regurgitation by Dye Dilution Curves}

Much attention has been given to the use of dye dilution curves in the assessment of valvular regurgitation since the observations of Korner and Shillingford (1955) that valvular regurgitation results in a lower peak concentration and a more gradual disappearance slope of the dye curve than would be expected. Attempts to measure regurgitant flow by this method have been unsatisfactory, however. The form of dye dilution curves is affected by many factors, including the cardiac output, velocity of the circulation, site of injection, and the size of various cardiac chambers, particularly the size of the left atrium in the case of mitral regurgitation.

Fig. 8 shows the disappearance slopes of the dye dilution curves of the patients in the present study, expressed as the number of seconds required for a tenfold drop in concentration on the downslope, when extrapolated as a straight line on semilogarithmic paper. This measurement is thus independent of absolute units of concentration. More complicated indices, involving peak concentration, appearance time, central volume, cardiac output, etc. have not proved more useful than this simple expression of the downslope. With this and other indices expressing similar aspects of the shape of dye dilution curves there is considerable overlap among patients with and

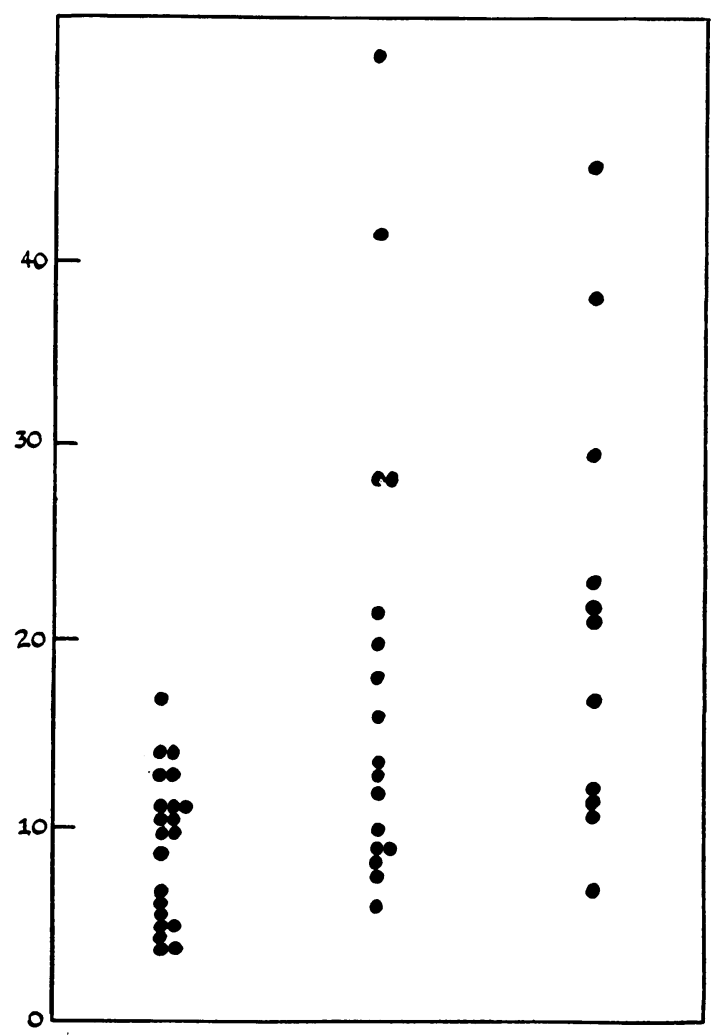

FIG. 8.-Frequency distribution of the downslope of the dye dilution curve, expressed as the number of seconds required for a tenfold fall in concentration. Pure mitral stenosis, left; combined stenosis and regurgitation, centre; pure mitral regurgitation, right. 
without regurgitation. The shape of the curve may be valuable evidence in assessing the degree of regurgitation, but is not necessarily reliable in an individual case.

The downslope of the dye dilution curve is systematically steeper in patients with pure mitral stenosis than in those with mitral regurgitation. There is, however, no systematic difference between patients with pure regurgitation and those with combined stenosis and regurgitation, although the majority of the latter have predominant stenosis. In other words, in a patient with mitral regurgitation, the contour of the dye curve has little or no correlation with the presence or absence of significant mitral stenosis in that patient. The data in Fig. 8 might suggest that mitral regurgitant flow is as large on the whole when there is associated stenosis of marked degree as it is in pure mitral regurgitation. On the other hand, it may be that the shape of the dye dilution curve is reflecting primarily the degree of left atrial enlargement, which tends to be greater in mitral regurgitation, with or without stenosis, than in pure stenosis.

\section{Special Features of Pure Mitral Regurgitation}

Left heart catheterization has made it possible to define the clinical entity of pure mitral regurgitation on a more objective basis than was previously possible-namely, the entity of mitral regurgitation with no significant diastolic pressure gradient across the valve. Excluding those with aortic valve disease and those with regurgitation that was surgically induced, there were 11 such patients. Most were advanced cases with much cardiac enlargement, and chronic or recurrent congestive heart failure. Eight had atrial fibrillation. The characteristic physical signs included a normal or soft first heart sound at the apex, a loud apical holosystolic murmur, and an apical diastolic rumbling murmur, soft and diminuendo but often of considerable length. An opening snap was present in only one, and that possibly tricuspid rather than mitral in origin, as a small pressure gradient was demonstrated across the tricuspid valve. Electrocardiograms usually showed low voltage, an intermediate or horizontal QRS axis, and some evidence of both right and left ventricular hypertrophy in the præcordial leads. Right ventricular hypertrophy was doubtless due to the pulmonary hypertension which was significant in six of the seven in whom right heart catheterization was carried out. The pulmonary vascular resistance ranged up to 8.5 units. The systemic cardiac index ranged from 1.6 to $4.31 . / \mathrm{min} . / \mathrm{m}^{2}$. The three patients with normal rhythm all had P mitrale, but did not have giant $a$ waves in the left atrial pulse form and did not have crescendo presystolic murmurs.

These patients were, of course, selected for study because it was suspected that important mitral stenosis might be present. Patients with more classical signs of pure mitral regurgitation, e.g. a loud systolic murmur only with a third heart sound and left ventricular enlargement, have not been studied.

\section{Mitral Stenosis of no Physiological Significance}

As in aortic stenosis, the clinical signs of mitral stenosis may be present when the lesion is so mild as to be undetectable by direct physiological assessment. Two patients have been seen with clinical signs of pure mitral stenosis, in whom no significant mitral diastolic pressure gradient could be demonstrated. Clinical features in the two patients were similar. Both were women 50 years of age, who had atrial fibrillation, and who complained in a neurotic manner of palpitation and to a lesser extent of dyspnœa and fatigue. Both had a faint but prolonged mitral diastolic rumbling murmur, and one had a loud opening snap of the mitral valve (Fig. 9). Both had normal heart size, but slight left atrial enlargement. The cardiograms were normal except for atrial fibrillation. An obstructive lesion having been ruled out, it was concluded that there was only mitral valvulitis of little or no functional significance, and that symptoms were due to atrial fibrillation and psychoneurosis, and possibly myocardial insufficiency. The left ventricular end-diastolic pressure was normal in one $(5 \mathrm{~mm} . \mathrm{Hg})$ and slightly raised in the other $(13 \mathrm{~mm} . \mathrm{Hg})$.

2D 
Fig. 9.-(A) Consecutive left atrial and left ventricular pressures in a patient with clinical signs of mitral stenosis but no significant pressure gradient across the valve. (B) Phonocardiogram of the same patient, showing the opening snap of the mitral valve.

\section{The Murmur of Mitral Stenosis}

It has already been mentioned that a long rumbling mitral diastolic murmur may be present in the absence of a significant pressure gradient across the valve, with or without associated regurgitation. There is only a rough correlation between the loudness of the rumble and the size of the gradient. Five patients have been seen with no audible diastolic murmur in spite of a large gradient. In three of these, mitral stenosis was an incidental discovery during left heart catheterization for the investigation of aortic stenosis. Two of those with no murmur had atrial fibrillation, pulmonary hypertension, and congestive heart failure, circumstances in which disappearance of the murmur of mitral stenosis is well-recognized. In two, with normal rhythm and associated aortic stenosis, short presystolic murmurs were perhaps obscured by the loud first sound and aortic systolic murmur following. The fifth had pure mitral stenosis, in normal rhythm without heart failure, but with pulmonary hypertension.

The long recognized influence of cardiac rhythm on the murmur of mitral stenosis is well documented by pressure records. In normal rhythm, with a crescendo presystolic murmur, atrial contraction produces a giant $a$ wave in the left atrium, and the gradient is abruptly increased in the immediate presystolic period (Fig. 1). In atrial fibrillation, with a long diminuendo diastolic rumble, the gradient is always largest at the beginning of diastole, and diminishes steadily to its smallest value in end-diastole (Fig. 2). It has been difficult to correlate the length of murmurs as heard or recorded phonocardiographically with the presence or absence of a gradient persisting throughout diastole. In several cases the murmur has seemed clearly to end before the first sound, yet a large gradient was present throughout diastole. Likewise, in patients with normal rhythm and showing only a short presystolic murmur, the gradient is not merely presystolic, but is substantial throughout the diastolic filling period. 


\section{Myocardial failure in Mitral Valve Disease}

The importance of myocardial function as opposed to the mechanical obstructive factor in mitral valve disease has been controversial for a long time. Cardiologists generally agree that myocardial failure is an important factor in many cases, but specific proof of this is difficult to obtain. It was hoped that measurement of the left ventricular diastolic filling pressure by left heart catheterization might enable differentiation of patients suffering primarily from mechanically obstructive mitral stenosis from those suffering primarily from rheumatic heart disease with myocardial failure, in which case the left ventricular diastolic filling pressure might be raised. Studies of patients with aortic valve disease have often shown a gross rise of filling pressure in the left ventricle, and the pulmonary wedge pressure may be grossly raised in patients with heart failure due to hypertension, coronary artery disease, etc. In the present study, however, no patient with mitral stenosis has had an end-diastolic pressure in the left ventricle higher than $14 \mathrm{~mm}$. $\mathrm{Hg}$, except one who also had severe aortic stenosis (Fig. 10). Only one patient with pure mitral regurgitation without significant aortic valve disease had much rise of left ventricular filling pressure. The differences between the three groups do suggest that, on the whole, mitral regurgitation is associated with higher filling pressures in the left ventricle, and hence with left ventricular failure. However, the rise of left atrial pressure

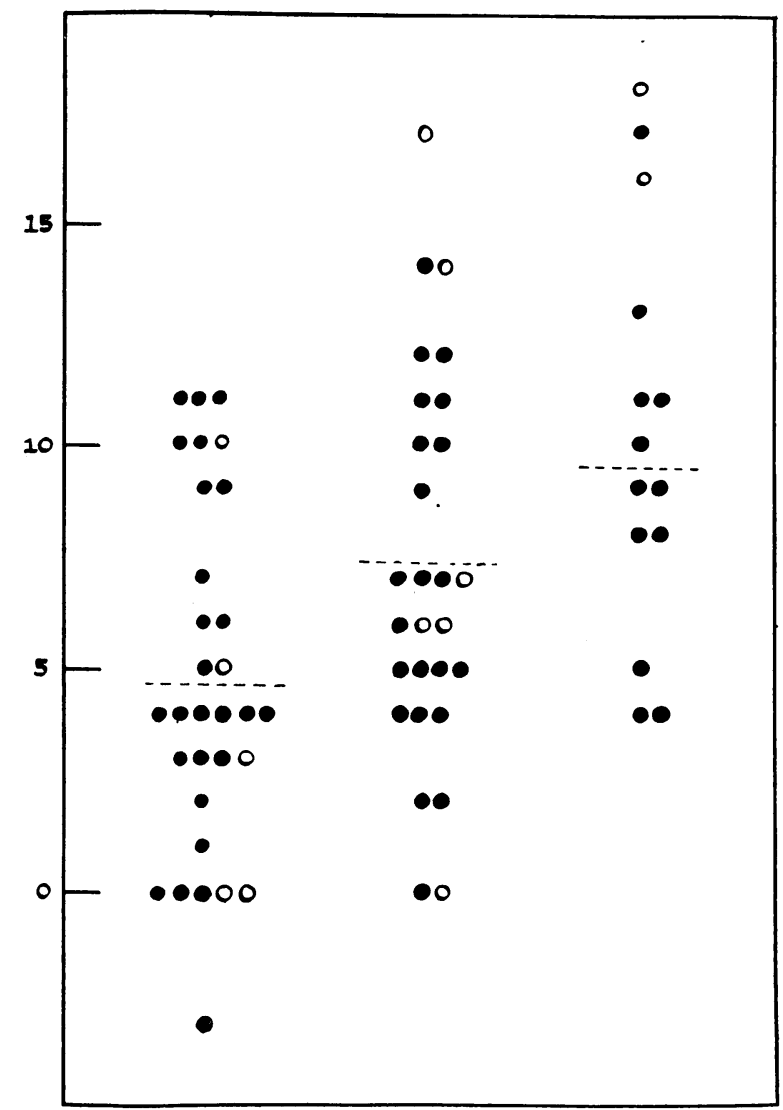

FIG. 10.-Frequency distribution of the left ventricular enddiastolic pressure, in $\mathrm{mm}$. $\mathrm{Hg}$ referred to mid-thoracic level. Pure mitral stenosis, left; combined stenosis and regurgitation, centre; pure mitral regurgitation, right. Solid circles represent patients without aortic valve disease. Open circles represent patients with significant aortic stenosis as well as mitral valve disease. 
in mitral regurgitation is generally not due to this, but rather (1) to the diastolic pressure gradient across the valve resulting from the associated stenosis, or (2) to the $v$ wave in systole, which ranges up to $65 \mathrm{~mm} . \mathrm{Hg}$ in height.

Cardiac failure was always treated first, and left heart catheterization only undertaken when the signs and symptoms of congestion were controlled as well as possible. Very few patients were studied while in a manifest state of congestive heart failure, and this fact may have been the cause of failure to demonstrate rises of left ventricular diastolic pressure. It appears, nevertheless, that left ventricular myocardial failure in patients with mechanically significant mitral stenosis is uncommon.

\section{Summary AND CONCLUSIONS}

Left heart catheterization by the Biork technique has been carried out in 75 patients with mitral valve disease. There have been no deaths or instances of cardiac tamponade due to the procedure. Hæmoptysis and pneumothorax occurred in 12 per cent, but less frequently with experience. Delayed arterial embolism occurred twice. The left atrium was successfully punctured in every case, and the left ventricle successfully catheterized in 96 per cent.

In pure mitral stenosis the severity of the lesion may be accurately assessed if the pressure gradient across the valve and the cardiac output are determined together. Clinical signs of mitral stenosis may be present in the absence of a significant pressure gradient. Five patients had severe mitral stenosis in the absence of a diastolic murmur.

In pure mitral regurgitation there is no significant pressure gradient across the valve in diastole. These patients cannot always be distinguished from those with predominant mitral stenosis by the clinical signs. Diastolic murmurs and pulmonary hypertension are commonly present.

In combinations of mitral stenosis and regurgitation, large diastolic pressure gradients are commonly present, even when regurgitation is the dominant lesion. Exact assessment of the valve size in this group is limited by inability to measure regurgitant flow. Estimation of a minimal valve area is of considerable value, and further information is gained from the left atrial pulse form and from dye dilution curves.

Left heart catheterization is a practical and useful diagnostic procedure in patients with mitral valve disease in whom clinical assessment is difficult.

Surgical observations were made by Dr. D. E. Harken, Dr. G. W. B. Starkey, Dr. J. S. Strieder, Dr. I. Madoff, Mr. R. H. S. Brain, Mr. D. N. Ross, and Sir Russell Brock. studies.

Dr. W. H. Abelmann, Dr. W. M. Madison, Jr., Dr. M. H. Proctor, and Dr. A. M. Johnson collaborated in these

The guidance of Dr. Laurence B. Ellis and Dr. Charles Baker throughout this work is gratefully acknowledged.

\section{REFERENCES}

Biork, V. O., Malmstrom, G., and Uggula, L. G. (1953). Ann. Surg., 138, 718.

Gorlin, R., and Gorlin, S. G. (1951). Amer. Heart J., 41, 1.

Gunning, A. J., and Linden, R. J. (1958). Circulation, 17, 354.

Korner, P. I., and Shillingford, J. P. (1955). Clin. Sci., 14, 553.

Marshall, H., Connally, D. C., and Wood, E. H. (1957). Circulation, 16, 913.

Morrow, A. G., Braunwald, E., Haller, J. A., and Sharp, E. H. (1957). Circulation, 16, 399.

Owen, S. G., and Wood, P. (1955). Brit. Heart J., 17, 41.

Shillingford, J. P. (1958). Brit. Heart J., 20, 229.

Woodward, E., Burchell, H. B., and Wood, E. H. (1957). Proc. Staff Meetings Mayo Clinic, $32,518$. 